\title{
A Randomized controlled trials on the effects of Acupuncture and Proprioceptive Neuromuscular facilitation (PNF) on supraspinatus tendinitis
}

\author{
*Sokunbi OG (PhD), Muhwhati, L (MSc), Robinson, P (BSc) \\ ${ }^{I}$ Department of Medical Rehabilitation, College of Medical Sciences University of Maiduguri, Nigeria \\ ${ }^{2,3}$ Occupational Health Advisor, Cummins Engine, Daventry, UK
}

\begin{abstract}
:
Objective of the study: This randomized controlled trial was undertaken to evaluate the effectiveness of acupuncture and Proprioceptive neuromuscular facilitation (PNF) as a treatment for supraspinatus tendinitis.

Methods: Thirty-five patients with a diagnosis of supraspinatus tendinitis were randomly allocated to either to a PNF group or a PNF plus acupuncture group and treated twice weekly for a period of 6 weeks. Functional mobility, power, and pain were assessed by a blinded assessor using the Constant Shoulder Assessment, at baseline, 6 weeks and 20 weeks. Analysis was based on the intention-to-treat principle.

Results: Both the PNF exercises only and the PNF with acupuncture groups experienced significant improvement at the end of 6 week treatment. Compared with the PNF group, the PNF plus acupuncture group experienced significantly greater improvement with treatment. Improvements in scores by $39.8 \%$ (standard deviation, 27.1) and 76.4\% (55.0) were seen for the PNF and the PNF plus acupuncture groups, respectively at 6 weeks $(P=0.048)$, and were sustained at the 20-week re-assessment $(40.3 \%$ [26.7] and 77.2\% [54.0], respectively; $P=0.025$ ).
\end{abstract}

Conclusion: We conclude that the combination of acupuncture with PNF shoulder exercise may offer effective treatment for supraspinatus tendinitis.

Key Words: Shoulder, Supraspinatus tendinitis, acupuncture, PNF, Physiotherapy

\section{Introduction and Review of Literature}

Supraspinatus tendinitis is the commonest lesion at the shoulder and is marked by painful resisted abduction (1). Often it shows no tendency to spontaneous recovery. Patients with Supraspinatus Tendinitis present with shoulder pain, movement restriction decrease functional activity.

The pain is not usually due to acute trauma or a single event. The supraspinatus is a muscle that originates in the supraspinatus fossa of the scapula, runs over the humeral head and attaches to the superior head of the greater tubercle of the humerus. The attachment of the superior aspect of the grater tubercle has a poor blood supply that makes it prone to injuries, and for this reason it is called "critical zone" (2). Repetitive use compression and subacute straining of this anatomical region causes microtrauma and as a consequence inflammation of the tendon. When the supraspinatus gets injured, its antagonist (latissimus dorsi, levator scapulae, rhomboids and pectoralis) lock the glenohumeral joint causing decrease scapular elevation and of scapulohumeral rhythm. When supraspinatus muscles get inflamed, it swells and increases size making difficult for it to glide in the ring formed from the bony and ligamentous coracoacromial arch over the scapula (3).

The site of the lesion can lie at any one of the four different sites which could be readily differentiated by the presence or absence of a painful arc and/or pain on full elevation (1).

I) The tenoperiosteal site: this site of lesion is characterized by painful restricted abduction accompanying by a painful arc which also shows that the lesion is in a pinchable position between the acromion and the greater tuberosity (1).Thus momentary pain is caused as the inflamed area squeezes under the acromial arc on arm elevation and if the arc is more pronounced when the arm is raised palm upwards, the lesion lies at the anterior aspects of the tenoperiosteal junction. In the palm downward is the more painful, this points to the posterior aspect of the tendinous insertion (1).

II) The deep aspect of tenoperiosteal junction: with this site of lesion, painful resisted abduction linked with pain on full passive elevation shows the lesion is where the grater tuberosity and the glenoid rim pinch the tendon at the deep aspect of the tenoperriosteal junction.

III) The distal end of the tendon: a painful resisted abduction accompanied by both painful arc and pain on full elevation indicates that the lesion occupies the distal end of both superficial and deep aspect of the tendon 
IV) The musculotendinous junction: if resisted abduction hurts with neither a painful arc on full elevation, attention is drawn to the proximal end of the tendon, i.e. the musculotendinous junction

Often the shoulder pain could be due to calcific tendonitis of the supraspinatus. The calcification can be caused by two factors. The first is a long standing impingement that causes tendinous fiber degeneration in the beginning and calcification later. The second is the reduced blood flow in the area that leads to calcification. In general it has been suggested that the calcification process starts with necrosis of the tendon with consequent formation of fibrocartilagenous mass, later calcium salts (hydroxyapatyte) will deposit on the aforementioned mass forming the calcification. The poor blood supply of the distal portion of the supraspinatus, the critical zone, is due to continuous pressure of the humeral head on the tendon when the arm is abducted (4).

The primary function of the supraspinatus tendon is abduction of the shoulder. When the supraspinatus is weak, the humeral head doesn't slide properly in the glenoid cavity and is in a position higher than normal. This mechanical problem could cause bursitis and subacromial impingement. The proper functioning of the supraspinatus and rotator cuff in general will be restored only after strengthening of the supraspinatus. It is important to treat supraspinatus tendonitis as soon as possible to avoid complications such as frozen shoulder, bursitis and permanent loss of function. Many options are available in the treatment of the supraspinatus tendinitis and supraspinatus tear, from conservative to invasive such as injections and surgery. In many cases supraspinatus surgeries are not successful, so all possible conservative approaches should be tried before surgery. Acupuncture has been reported to be effective for the treatment of supraspinatus tendinitis. However, it seems that not many randomized controlled trials have been carried out to validate this claim. Consequently, we designed the current randomized, single-blind controlled clinical trial to investigate the effects of Proprioceptive neuromuscular facilitation exercise and acupuncture treatment, based on the principles of TCM, as a treatment for supraspinatus tendinitis.

\section{Ethical approval}

\section{Methods} Daventry,UK.

The study was approved by the Research Ethics Committee Occupational Health Unit Cummins Inc

\section{Subjects}

The patients selected for inclusion were consecutive sample of men and women attending the occupational health department of Cummins Inc Daventry, UK Clinic following doctors' referral, with a diagnosis of supraspinatus tendinitis. The diagnosis of supraspinatus tendinitis was based on a history of limited motion of the glenohumeral joint, with painful restricted abduction (1). In most cases, the onset was spontaneous and gradual, while in some it was precipitated by relatively minor trauma.

\section{Inclusion criteria}

1. Shoulder pain with painful restricted shoulder mobility for at least 1 month and less than 12 months' duration;

2. Appreciable restriction of both active and passive motion with abduction and flexion not exceeding

$90^{\circ}$ and external rotation not exceeding $30^{\circ}$ and with or without

3. Pain at night

\section{Exclusion criteria}

1. History of major shoulder injury or surgery;

2. Clinical or radiological evidence of other pathology that could possibly account for symptoms;

3. Patients with evidence of cervical radiculopathy, paresis, or other neurological changes in the upper limb on the involved side;

4. The presence of underlying fracture, associated inflammatory arthritis, known renal or hepatic disease, haemopoietic disorder, malignancy, any mental disorder likely to interfere with the course or assessment of the disease process

\section{Randomization into intervention groups}

Patients fulfilling these criteria were asked to participate in the trial. The aim and method of the study was explained to them. After obtaining their informed consent, the patients were randomly allocated to one of the two treatment groups, using the random table generated by Microsoft excel.

The patients were instructed to use the shoulder and arm normally, but within the limits of their pain. Patients who received other types of therapy during the study or failed to attend scheduled follow-up for treatment and assessment were excluded in the final analysis. The management regimen for the two treatment groups is described below. 


\section{Interventions}

\section{PNF Exercise group}

The patients in this group received physiotherapy in the form of a standard PNF exercise programme led by the same physiotherapist each session. Treatment was scheduled twice a week for a period of 6 weeks, and each treatment session lasted for 30 minutes. Careful instructions were given to the patients when demonstrating exercises. The PNF exercise protocol used were similar to those previously reported by (5). Four different patterns of PNF were performed are as follows: - D1 Flexion pattern: shoulder flexion, elbow flexion, forearm supination, wrist flexion, and finger flexion.

- D1 Extension pattern: shoulder extension, elbow extension, forearm pronation, wrist and finger extension. - D2 Flexion pattern: shoulder flexion, elbow extension, forearm supination, wrist extension, and finger extension.

- D2 Extension pattern: shoulder extension, elbow flexion, forearm pronation, wrist and finger flexion.

In addition to PNF patient were also instructed in gentle stretching exercises, including stretching the shoulder in external rotation, internal rotation, cross-body adduction, and forward flexion, which did not engender substantial shoulder pain were carried out (see Appendix I). Exercises with the arm in more than $40 \square$ of flexion or abduction were to be undertaken with caution (1). Patients were instructed to perform this designated types of shoulder exercise 10 times twice daily at home during the trial period. Home exercise monitoring cards were given to patients for record purposes. Compliance with the home exercise programme was checked by an independent assessor, using the home exercise monitoring record card.

\section{PNF Exercise plus Acupuncture group}

Both the PNF exercise and home exercise programme were conducted in the same way as for the exercise group. The therapist was blinded to the two groups of patients. In addition, acupuncture was administered twice a week for a period of 6 weeks by physiotherapist, using classical Chinese acupuncture. The physiotherapist administering acupuncture was a well experienced physiotherapist certified by acupuncture association of chartered physiotherapist, UK to carry out acupuncture treatment.

Patient preparation

The patient was placed in a sitting position and the skin over the acupoint was sterilized with $75 \%$ alcohol. A sterilized $7.62 \mathrm{~cm}$ (3 inches) long, 30 gauge, disposable,

\section{Needling technique}

Ipsilateral needling was adopted, that is, the right-side acupoint was used for right side of the affected shoulder (6) . In patients with supraspinatus tendinitis pain is typically presented in the middle of the shoulder, thus a local and distal points of Sanjio channels were selected. If a patient is presented with pain in the anterior side of the shoulder, points on the large intestine (LI.15, 16) were added together with a distal point of this channel (LI.4, 11). If pain is located on the posterior side of the shoulder joint, local point on small intestine channel (SI.9-11) were used in combination with distal point of this channel SI.6 (Stux and Pomeranz, 1998). Needles were inserted perpendicular to each of the points stated above to a depth of about $6.35 \mathrm{~cm}$ ( 2.5 inches). This was followed by strong stimulation from wide amplitude needle rotation simultaneously with lifting and thrusting movements, to evoke a marked needling sensation (de qi), commonly described as tingling, numbness, soreness, dull pain, heaviness, or distention at the site of the needle insertion. The needle was retained for 20 minutes, with three 1-minute needle manipulations made over this period.

Functional mobility, power, and pain were assessed by a blinded assessor using the Constant Shoulder Assessment, (CSA), [See appendix II]. Reliability and concurrent validity of the constant shoulder assessment has been reported (7). Assessment was completed prior to the commencement of treatment (baseline) and at the end of the 6th week (at the completion of treatment). Follow-up assessment was completed at the end of the 16 week. The CSA has a maximum score of 100 points, with subjective and objective components included in a ratio of 35:65. Subjective parameters assess the degree of pain the patient experiences and his or her ability to perform normal tasks of daily living described in both activity- and position-related terms. These parameters are assessed independently before objective testing of active motion range and shoulder power is completed. The objective parameter of active motion range is based on the active range of composite movements that allow the placement of the upper limb in functionally relevant positions, with a goniometer to measure forward and lateral elevation, and positioning of the hand in relation to the head and trunk for assessment of rotation. Scoring of shoulder power is based on the number of pounds of pull the patient can resist in abduction, up to a maximum of $90 \square$. The score given fornormal power is 25 points, with less given proportionately for less power. A total CSA score of 100 points indicates perfect, pain-free movement and function.
} 


\section{Sample Size Calculation}

An earlier sample size calculation indicated a minimum of 13 patients should be recruited in each group, based on a 75\% improvement in CSA with a 5\% chance of committing a false positive error and $80 \%$ power (Cohen 1988).

\section{Data analysis}

Statistical analysis was conducted based on the intention-to-treat principle. Specifically, all recruited patients were analysed as randomized regardless of the actual treatment received or whether they withdrew before the end of follow-up. Missing values were imputed by the last available observation of the corresponding patients. To determine the robustness of conclusions, the analysis was repeated when missing data were discarded. Comparison of the two groups at baseline (on admission) was first made using the $\square \mathbf{2}$-test and Mann-Whitney $U$ test according to whether the variable under consideration was categorical or continuous, respectively. Then the difference in mean CSA scores within each group was evaluated using Friedman's test. Mean CSA scores and percentage of CSA improvement from baseline at each measurement visit were compared between the two groups by Mann-Whitney $U$ test. The P value of each significance test was determined exactly or approximated by Monte Carlo simulation. The Statistical Analysis System (Windows version 8.1; SAS Institute Inc., Cary, NC, US) was used for all statistical analysis.

\section{Results}

Consecutive samples of 17 patients were randomized and allocated to the PNF exercise group and 18 patients to the PNF exercise plus acupuncture group. No patient received acupuncture as treatment prior to the study. One patient in the exercise plus acupuncture group discontinued treatment after the second acupuncture session due to fear of needle pain, while four patients withdrew from the exercise group after 6 weeks of exercise practice without given any reason for doing so. Nevertheless, all patients were considered in the subsequent analysis based on the intention-to-treat analysis. Admission data for the patients is summarized in table 1. There were no statistically significant differences between the two groups in terms of age, sex, and duration of symptoms. There were no statistically significant differences between the two groups with respect to the amount of analgesic intake before, during, and after treatment (Mann-Whitney $U$ Test: baseline, $\mathrm{P}=0.573 ; 6$ week, $\mathrm{P}=0.768 ; 20$-week, $\mathrm{P}=0.921$ ) [Fig 3]. No patient took analgesic therapy other than ibuprofen, or received other types of therapy for the shoulder pain during the study period.

The mean CSA scores for both groups are shown in Table 2. There was no statistically significant difference between the groups in terms of their baseline CSA scores $(\mathrm{P}=0.981)$ and CSA scores at 6 weeks $(\mathrm{P}=0.06)$. The CSA scores, however, were significantly higher in the PNF exercise plus acupuncture group compared with the exercise group at 20 weeks $(\mathrm{P}=0.04)$. Within each group, there was a significant difference among mean CSA scores measured at baseline, 6 weeks, and 20 weeks ( $<<0.005$ by Friedman's test). The percentage of CSA improvement from baseline for each patient was computed and a summary is shown in Table 2. At 6-week assessment, there was a $86.7 \%$ and a $43.9 \%$ improvement in shoulder function for the exercise plus acupuncture group and the exercise group, respectively. These relative improvements were sustained at the 20 -week reassessment (87.9.2\% and $45.2 \%$ for the exercise plus acupuncture and exercise groups, respectively). Compared with the exercise group, the exercise plus acupuncture group was significantly better after treatment at 6 weeks and on follow-up at 20 weeks $(\mathrm{P}=0.04$ and $\mathrm{P}=0.00$ respectively). The analyses were repeated when all missing values were discarded. All conclusions were essentially identical with the exception of a significant difference in mean CSA scores now seen between the two groups at 6 weeks $(\mathrm{P}=0.03)$. Final conclusions were drawn, however, from the intention-to-treat analysis

\section{Discussion}

The outcome of this study has shown that acupuncture and PNF exercise is an effective treatment option that can enhance the speed and degree of recovery of supraspinatus tendinitis. The traditional Chinese medicine consider human body as an entity divided by a series of meridians or channels in which flow the energy, also referred as chi, blood and body fluids. Along these meridians are located the acupuncture points are cutaneous areas containing nerve endings, nerve bundle, lymphatic vessels and capillaries. When a needle is inserted in an acupuncture point, a stimulus is transmitted first to the spinal cord and then to the hypothalamus which releases neurotransmitters with opiate like activities like endorphin which reduces the pain intensity or caused the pain to disappear (8).

Although there have been several reports on the efficacy of acupuncture on supraspinatus tendinitis, convincing scientific evidence for its efficacy is still lacking $(9,10)$.Previous studies of acupuncture treatment provide equivocal results due to limitations in their design. The limitation ranges from lack of sensitive and valid outcome measure, the virtual impossibility of blinding the acupuncturist, the uncertainties in choosing a 
control acupuncture point, and the inherent difficulties in the use of appropriate controls, such as placebo and sham acupuncture groups $(9,10,11)$.

In the present study, CSA was used for evaluation of progress following treatment. This assessment is a simple clinical tool that combines functional assessment of the shoulder with assessment of individual parameters, such as pain and daily activity. It therefore allows evaluation of progress after injury, treatment, or disease with respect to these individual parameters or in terms of overall function. The CSA is easy to use, taking only a few minutes to perform. It is reliable and valid in the overall assessment of shoulder function, with low inter-observer and intra-observer error rates. There are, however, two limitations of the CSA Firstly, assessment of power is error-prone as accurate measurement of power is difficult to achieve. Shoulder movement is complex and consequently measurement of power in a single arc of shoulder movement is unlikely to be representative of full functional potential. Secondly, in cases of shoulder instability, such as joint dislocation, CSA fails to reflect accurately the true level of disability incurred and thus is a not a reliable outcome measure for patients with complaints of instability. Supraspinatus tendinitis is characterized by pain and limitation of motion without fracture and dislocation. Joint instability and marked power loss are rarely seen in these group of patients and thus, these limitations are not pertinent to the study population. The Constant Shoulder Assessment is, therefore, a good outcome measure to evaluate the severity.

Acupuncture treatment used in this clinical trial was conducted according to the principles of TCM.

Ancient Chinese medicine considers human health as facing the tensions created by opposing forces in naturethe Yin and the Yang. Medical intervention carried out according to this concept aims to restore balance between the opposing energy forces. A concept of vital energy flow linking circulation to neurological function is fundamental to the practice of acupuncture (9) The vital life energy, Qi, is thought to flow through a set of interconnected channels, called meridians, which follow a circadian rhythm. The meridians are interconnected by Qi. Each internal organ is thought to be associated with a certain meridian, and the meridian is named after the organ concerned. Diseases and discomforts, such as pain, are classified according to the meridians they involve, whether they have a Yin (cold, hypo functional) or Yang (hot, hyper functional) nature, and whether the flow of Qi is excessive or deficient. According to TCM, pain in the shoulder is associated with weakness in the 'stomach' and 'spleen', and deficiency of Qi. Supraspinatus tendinitis belongs to the group of diseases characterized by blockage of $\mathrm{Qi}$, or to the Bi syndrome, that is, painful locomotor disorders. The definition of $\mathrm{Bi}$ in Chinese medicine is obstruction or interference with the flow of Qi and blood. It is mainly believed to be due to the deficiency of Yin and to inadequate defence of the skin against invasion by the pathogenic factors of wind, cold, and dampness into the body. The resulting stasis of Qi and blood in the channels leads to pain, aching, and stiffness in the muscle, bones, tendons, and joints $(9,10)$. The present study, however, has shown that combination of acupuncture treatment and Proprioceptive neuromuscular facilitation could be useful to relieve menace of this particular musculoskeletal problem.

\section{Limitation}

There are a few limitations evident in this study. Due to the inherent difficulty in long-term studies of chronic pain, as discussed previously, follow-up of patients was for a maximum of 20 weeks. Long-term followup is necessary, however, in order to determine whether lasting benefits of acupuncture have occurred. Failure to undertake long-term follow-up has the potential to produce false-positive outcomes, that is, positive outcomes when no real treatment effect exists. The lack of a placebo or sham acupuncture control group in this clinical trial has made it impossible to prove whether needling was an important part of the method or whether the improvement felt by the patients in the exercise plus acupuncture group was due to the therapeutic setting and psychological phenomena.

\section{References}

[1] Cyriax J.H. (1983) Cyriax's illustrated Manual of Orthopaedic Medicine $2^{\text {nd }}$ Edition Butterworth, Heinemman $42-45$

[2] Linder H, (2000) Clinical Anatomy Appleton and Lange Norwalk California 525-526

[3] Jacobson, E. Lockwood, M. (1989), Shoulder pain and repetition strain injury to the supraspinatus muscle American Osteopathic Association 98 (8) 1037-1045

[4] Harmeyer R.. (1995) Chiropractic Rehabilitation of the Frozen shoulder Journal of the American Chiropractic Association 32(7) 596012

[5] DeBerardino T. (2013) Supraspinatus Tendinitis Treatment \& Management. Medscape Reference. http://www.emedicine.medscape.com/article/93095-treatment

[6] Bradnam, L. (2007). A proposed clinical reasoning model for western acupuncture, Journal of the Acupuncture Association of Chartered Physiotherapists, 21-30.

[7] Constant CR, Murley AH.(1987) A clinical method of functional assessment of the shoulder. Clin Orthop ;214:160-4

[8] Gellman H (2002) Acupuncture Treatment for musculoskeletal pain Acupressure techniques Healing Arts Press 7-8

[9] Ezzo J, Berman B, Hadhazy VA, Jadad AR, Lao L, Singh BB (2000). Is acupuncture effective for the treatment of chronic pain? A systemic review. Pain ;86:217-25

[10] Lee TL. (2000) Acupuncture and chronic pain management. Ann Acad Med Singapore ;29:17-21.

[11] Moore ME, Berk SN (1976). Acupuncture for chronic shoulder pain. An experimental study with attention to the role of placebo and hypnotic susceptibility. Ann Intern Med 1976;84:381-4. 
Table 1: Baseline data of patients

\begin{tabular}{|l|l|l|l|}
\hline Baseline Characteristics & PNF Exercise Group & $\begin{array}{l}\text { PNF Exercise } \\
\text { Acupuncture Group }\end{array}$ & \&-values \\
\hline No. of patients & 17 & 18 & 0.7 \\
Sex ratio (M:F) & $9: 8$ & $42.9(8.5)$ & $0.73^{*}$ \\
Age & $45.5(6.7)$ & $7.3(5.9)$ & $0.57 \dagger$ \\
Mean(SD) & & & $1.00 \dagger$ \\
Duration of symptoms (months) & & & \\
Mean(SD) & & & \\
\hline
\end{tabular}

\section{$\uparrow$ Mann-Whitney $\boldsymbol{U}$ test}

Table 2: Constant Shoulder Assessment scores and percentage improvement with treatment

\begin{tabular}{|c|c|c|c|}
\hline Constant shoulder assessment scores & $\begin{array}{l}\text { PNF Exercise Group } \\
\text { Mean (SD) }\end{array}$ & $\begin{array}{l}\text { PNF Exercise \& } \\
\text { Acupuncture Group } \\
\text { Mean (SD) }\end{array}$ & $\begin{array}{l}\text { Between group } \\
\text { difference } \\
\text { P-values }\end{array}$ \\
\hline Baseline & $50.8(12.3)$ & $52.6(10.7)$ & 0.98 \\
\hline 6 weeks & $55.2(15.0)$ & $75.3(13.5)$ & 0.06 \\
\hline 20 weeks & $55.9(10.5)$ & $75.9\left(\begin{array}{ll}1 & 8.5\end{array}\right)$ & $0.04 *$ \\
\hline $\begin{array}{l}\text { Non-parametric tests (Friedman's ANOVA by } \\
\text { ranks) comparing the } \\
\text { Constant Shoulder Assessment scores } \\
\text { at the three different times of assessment }\end{array}$ & $\mathrm{P}<0.005$ & $\mathrm{P}<0.005$ & - \\
\hline $\begin{array}{l}\text { Percentage improvement from baseline } \\
\text { At } 6 \text { week } \\
\text { At } 20 \text { week }\end{array}$ & $\begin{array}{l}43.9 \%(23.7 \%) \\
45.2 \%(19.8 \%)\end{array}$ & $\begin{array}{l}86.7 \%(30.7 \%) \\
87.9 \%(20.7 \%)\end{array}$ & $\begin{array}{l}0.04 * \\
0.00 *\end{array}$ \\
\hline
\end{tabular}

*Statistically significant
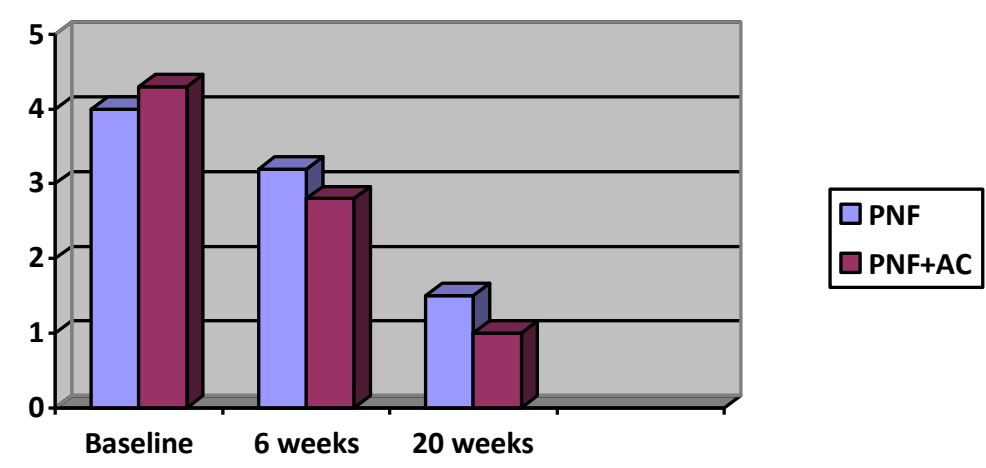

Figure 1: Analgesic intake (Ibuprofen capsule200mg/d)/week by patients receiving treatment for frozen shoulder

\section{Appendix 1: Stretching Exercises}

Stretching exercises should be done by repeating the exercise 3 times and holding the stretch each time for 30 seconds.:

i. Pendulum Swings

Stand and lean over with arm hanging. Begin swinging the arm in small circles and gradually enlarge the size of the circles. Repeat 10 times.

ii. Rotator Cuff Stretch I

Stand or sit.

Bend elbow at 90-degree angle, keeping elbows close to body, lower arms are pointed forward and parallel to floor, thumbs pointed up. 
Move hands away from body until stretch is felt in shoulder.

Hold stretch for 30 seconds. Rest and repeat.

iii. Rotator Cuff stretch II

Stand in front of doorframe bend elbow at 90-degree angle and place palm against doorframe.

Move forward to stretch rotator cuff.

iv. Rotator Cuff Stretch III

Sit or stand. Hands on waist, thumbs facing frontward. Bend at hips until body is resting on lap if sitting or parallel to floor if standing. Let arms hang toward floor to stretch the shoulder joint. Hold stretch for 20 seconds.

v. Back of shoulder stretch

Reach arm right across chest, use left hand to grasp arm just above elbow and gently pull arm farther across body until you feel a stretch in the back of the shoulder.

Hold stretch for 30 seconds. Rest and repeat.

Repeat on other side.

vi. Overhead Stretch

Place hands on edge of counter top.

Bend upper body at hips at 90 -degree angle.

Reach and stretch shoulders.

vii. Towel Stretch - Internal Rotation

Place right hand behind back.

With the left hand, dangle a towel behind the back.

Grasp the towel with the right hand.

Gently pull the right hand upward by raising the left arm to stretch the right shoulder.

Towel should be in vertical position.

Hold for 30 seconds. Repeat on other side.

viii. Towel Stretch - External Rotation

Place right hand behind back.

With the left hand, dangle a towel behind the back.

Grasp the towel with the right hand.

Pull right hand downward to stretch the left shoulder.

Towel should be in vertical position.

Hold for 30 seconds. Repeat on other side.

\section{Appendix II}

Constant Shoulder Assessment scale

\begin{tabular}{|l|l|l|}
\hline \multicolumn{1}{|c|}{ Assessment parameters } & \multicolumn{1}{|c|}{ Extent / Position } & Points \\
\hline Scoring for pain (maximum=15) & None & 15 \\
& Mild 10 & 10 \\
& Moderate & 0 \\
& Severe & \\
\hline $\begin{array}{l}\text { Scoring for activities of daily living level } \\
\text { (maximum=20) }\end{array}$ & Activity level & 4 \\
& Full work & 2 \\
& Full recreation/sport & 2 \\
& Unaffected sleep & 4 \\
& & 6 \\
& Positioning & 10 \\
& Up to the waist & \\
& Up to the xiphoid & \\
& Up to the neck & \\
& Up to the top of the head & \\
& Above the head & \\
\hline
\end{tabular}

